of years from $I_{n}$ corresponding to $n$ years. Various assumptions regarding the frequency distribution of the precipitation lead to corresponding sets of theoretical factors for standardizing the precipitation ratio and certain of these agree well with observations. Results plotted on coordinate paper having a reciprocal scale for $I_{n}$ and a logarithmic scale for $n$ facilitate a graphical treatment of the observations since the graph approximates to a straight line. The standard error of $I_{n}$ is also computed corresponding to the two most important frequency distributions of the precipitation. In order to reduce the accidental fluctuations of $I_{n}$ it is sometimes desirable to use the difference between the averages of the $r n$ highest and the $r n$ lowest values, where $r n$ equals some appropriate value, say 3 . Theoretical factors derived for correcting such values to obtain $I_{n}$ agree well with observations. (Received October $3,1940$.

78. Jerzy Neyman: A statistical problem in mass production and routine analyses.

Let $x_{i 1}, x_{i 2}, \cdots, x_{i n}$ denote the results of parallel analyses of some $i$ th sample, $i=1,2, \cdots, N$ (a sample of items of mass production). The $x$ 's are considered as random variables following the law $p\left(x_{i j}\right)=\left(\sigma_{i}(2 \pi)^{1 / 2}\right)^{-1} \exp \left\{\left(x_{i j}-\xi_{i}\right)^{2} / 2 \sigma^{2}{ }_{i}\right\}$. Denote by $H$ the hypothesis that $\sigma_{1}=\sigma_{2}=\cdots=\sigma_{N}$, that is, that the precision of measuring $\xi_{i}$ is maximum, and let $x_{i}=\left(\sum_{j} x_{i j}\right) / n, S_{i}^{2}=\sum_{j}\left(x_{i j}-x_{i}\right)^{2}, V=\sum_{i} S_{i}^{2}, U=\sum_{i} S_{i}^{4}$. Also let $E$ denote the observed point in the space $W$ of the $S_{i}^{2}$ s and $w(V)$ the part of the locus $V=$ const. included in any region $w \subset W$. It is proved that (1) a necessary and sufficient condition for a test's probability of rejecting $H$ when true to be a fixed value $\alpha$ is that its critical region $w=\sum_{v} w(V)$, with the $w(V)$ arbitrary except that $P\{E \in w(V) \mid E \in W(V)\}=\alpha$ for any $V>0$. (2) If $H$ is not true and $h=\sigma^{-2}$ is a random variable with $p(h)=c h^{\alpha-1} e^{-\beta h}$, then the critical region $w_{0}$ determined by $U>U_{\alpha} V^{2}$, with $P\left\{U>U_{\alpha} V^{2} \mid H, V\right\}=\alpha$, has the following useful properties: (a) if $H$ be true, then the probability of $w_{0}$ rejecting $H$ is equal to $\alpha$, (b) the first derivative of the power function of $w_{0}$, taken at the point of $H$ being true, is equal to zero, (c) the second derivative is maximum. ((b) is true for all regions satisfying (a).) (Received October 28, 1940.)

\title{
THEORY OF NUMBERS
}

\section{E. T. Bell: Selective equations.}

The symbols ()$^{\prime},[]^{\prime}$ denote the least, greatest of the integers occurring within the symbols. Each such integer may be replaced by a symbol ( )', [ ]' referring to a new set of integers, and so on, a finite number of times. The most general system of equations consisting of equalities between single power products formed from ( )', []$^{\prime}$ is solved non-tentatively and finitely, by passing to a unique dual of the system, in which ( ), the G.C.D., and [ ], the L.C.M., replace ( )', [ ]'. The latter system is a simple multiplicative system, and hence is completely solvable non-tentatively and finitely. The problem solved arose in the detailed discussion of compound multiplicative systems. (Received October 26, 1940.)

\section{Leonard Carlitz: Finite differences and polynomials in a Galois} field.

This paper is concerned chiefly with the solution of equations such as $\sum f(M) M(t)$ $=g(t)$ and $\sum f(M, u) M(t)=g(u, t)$, the summation extending over all polynomials $M=M(x)$ of degree less than $m$. (Received November 25, 1940.) 
81. Douglas Derry: On the boundary case of Minkowski's linear form theorem. Preliminary report.

Group theoretic methods are used to study Minkowski's conjecture regarding the boundary case of his linear form theorem, with the restriction that the coefficients become integral upon multiplication by a fixed prime $p$. In this case the validity of the conjecture is established for $n$ forms provided $p>(n-3) / 2$ and for all primes where $n \leqq 8$. (Received November 25, 1940.)

82. D. H. Lehmer : A general summation formula for numerical functions.

Let $h(n)$ be any single valued numerical function, and let $H(n)=h(1)+h(2)$ $+\cdots+h(n)$ be its sum. The author obtains a formula for $H(n)$ in terms of two functions $f(n), g(n)$ and their sum functions $F(n)$ and $G(n)$, such that $\sum f(\delta) g(n / \delta)$ $=h(n)$, the sum extending over the divisors of $n$. The formula involves a parameter $R$ which can be chosen at will. For $R=1$ or $R=n$ one obtains a formula used in several special cases by Dirichlet, while for $R=\left[n^{1 / 2}\right]$, a general formula of Franel and Glaisher is obtained, special cases of which have been used by many writers. By choosing $R$ an appropriate function of $n$ depending on $h$, the number of terms in the formula may be minimized. Exact formulas for the number of square-free numbers equal to or less than $x$ and the excess of numbers equal to or less than $x$ having an even number of prime factors over those having an odd number of prime factors are given as examples. In these formulas the number of terms varies as the cube root of $x$. (Received October 22, 1940.)

\section{Gordon Pall: Simultaneous representation in a quadratic and linear form.}

The problem of solving in integers $x_{i}$ the pair of equations $a=\sum c_{i} x_{i}^{2}, b=\sum c_{i} x_{i}$, where the $c_{i}, a$, and $b$ are given integers, and $t=\sum c_{i} \neq 0$, is reduced for certain forms of $t$ to the representation of $t a-b^{2}$ in a specified form in one fewer variables. When this form is in a genus of one class the solution is complete. Twenty-nine such sets $\left(c_{i}, \cdots\right.$, $c_{4}$ ) with positive $c_{i}$ are found. (Received November 25, 1940.)

84. R. M. Robinson: On the simultaneous approximation of two real numbers.

For each positive integer $s$, the smallest $C(s)$ is determined, such that for every pair of real numbers $\xi_{1}$ and $\xi_{2}$, there exist integers $a_{1}, a_{2}, b$ with $0<b \leqq s$ and $\left|b \xi_{k}-a_{k}\right|$ $\leqq C(s)(k=1,2)$. (Received September 28, 1940.)

85. J. B. Rosser: Explicit bounds for some functions of prime numbers.

In this paper the constants implicit in several "big $O$ " theorems of the analytical theory of primes are evaluated and improved. A typical result is the following. Let $\pi(x)$ denote the number of primes less than or equal to $x$. Then for $55 \leqq x, x /(\log x+2)$ $<\pi(x)<x /(\log x-4)$. (Received November 23, 1940.)

86. H. A. Simmons: Maximum numbers associated with a symmetric Diophantine equation in $n$ reciprocals.

In a previous paper (Duke Mathematical Journal, vol. 2 (1936), pp. 317-340) the 
writer excluded from consideration the equation $1 / x_{1}+1 / x_{2}+\cdots+1 / x_{n}+\lambda / x_{1} x_{2}$ $\cdots x_{n}=b /((c+1) b-1)$, in which $b, c$ are any positive integers, and $\lambda$ is an integer greater than 1 . The present paper identifies a class of maximum numbers relative to this equation. Using the terms $E$-solution and Kellogg solution, and the symbol $\Sigma_{n, r}(x)$ in senses previously employed, it is proved that if $w$ is the Kellogg solution and if $X \neq w$ is any $E$-solution of the equation, then $\Sigma_{n, n-1}(w)>\Sigma_{n, n-1}(X)$ and $\Sigma_{n, n}(w)>\Sigma_{n, n}(X)$. On account of these two inequalities, it is possible to associate with this equation a class of infinitely many maximum numbers. It is also shown that $w_{n}>X_{n}$, which might be called the Kellogg property. (Received October 17, 1940.)

87. L. I. Wade: Certain quantities transcendental over the field $G F\left(p^{n}, x\right)$.

In a previous paper (abstract 46-1-129) the author proved certain quantities transcendental over the field $G F\left(p^{n}, x\right)$. The present paper includes certain additional transcendental quantities. For example, $\sum_{k=1}^{\infty} 1 /\left(x^{p^{n k}}-x\right)$ is transcendental, $\sum_{k=0}^{\infty} 1 / x^{q^{k}}$ $(q>1)$ is algebraic if $q$ is of the form $p^{s}$ and transcendental otherwise, and $\sum_{k=0}^{\infty} 1 / x^{k^{q}}$ $(q>1)$ is transcendental. (Received November 25, 1940.)

\section{Max Zorn: Idempotency of infinite cardinals.}

This paper contains a simple proof of the theorem that the product of two infinite numbers is equal to the maximal factor. The application of ordinal numbers is replaced by the use of the maximum principle. (Received October 28, 1940.)

\section{Topology}

\section{Ben Dushnik and E. W. Miller: On the dimension of a partial} order.

Let $A$ be any set. Let $K$ be any collection of linear orders, each defined on all of $A$. A partial order $P$ on $A$ is defined as follows: For any two elements $a_{1}$ and $a_{2}$ of $A$, $a_{1}<a_{2}$ (in $P$ ) if an only if $a_{1}<a_{2}$ in every linear order of the collection $K$. A partial order so obtained will be said to be realized by the linear orders of $K$. With the aid of a result due to Szpilrajn on the linear extensions of a partial order (Fundamenta Mathematicae, vol. 16 (1930), pp. 386-389), it is seen that if $P$ is any partial order on a set $A$, then there exists a collection $K$ of linear orders on $A$ which realize $P$. By the dimension of a partial order $P$ defined on a set $A$ is meant the smallest cardinal number $m$ such that $P$ is realized by $m$ linear orders on $A$. It is shown that if $n$ is any natural number, there exists a finite partial order of dimension $n$, and if $m$ is any transfinite cardinal, there exists a partial order of dimension $m$ defined on a set of power $m$. Reversible partial orders (see abstract 46-5-266) are those of dimension not greater than 2. (Received October 25, 1940.)

\section{Samuel Eilenberg: Imbedding of spaces into euclidean spaces. Preliminary report.}

Given a metric space $X$, let $P(X)$ be the subset of the cartesian product $X \times X$ consisting of all points $(x, y) \in X \times X$ such that $x \neq y$. If every $i$-dimensional compact Vietoris cycle $\bmod 2$ of $P(X)$ bounds in $P(X)$ for $i=1,2, \cdots, n-1$, then $X$ is not imbeddable topologically into the euclidean $n$-space. Many other results of this type are obtained. Analogous theorems hold if the join $X \circ X$ is considered instead of the product $X \times X$. (Received November 25, 1940.) 